\title{
Modeling of Adaptive Optics-Based Free-Space Communications Systems
}

S.C. Wilks, J.R. Morris, J.M. Brase, S.S. Olivier, J.R. Henderson, C. Thompson, M. Kartz, A.J. Ruggerio

This article was submitted to SPIE Optical Science and Technology Conference, Seattle, WA, July 7-11, 2002

\section{August 6, 2002}

U.S. Department of Energy






\section{DISCLAIMER}

This document was prepared as an account of work sponsored by an agency of the United States Government. Neither the United States Government nor the University of California nor any of their employees, makes any warranty, express or implied, or assumes any legal liability or responsibility for the accuracy, completeness, or usefulness of any information, apparatus, product, or process disclosed, or represents that its use would not infringe privately owned rights. Reference herein to any specific commercial product, process, or service by trade name, trademark, manufacturer, or otherwise, does not necessarily constitute or imply its endorsement, recommendation, or favoring by the United States Government or the University of California. The views and opinions of authors expressed herein do not necessarily state or reflect those of the United States Government or the University of California, and shall not be used for advertising or product endorsement purposes.

This is a preprint of a paper intended for publication in a journal or proceedings. Since changes may be made before publication, this preprint is made available with the understanding that it will not be cited or reproduced without the permission of the author.

This work was performed under the auspices of the United States Department of Energy by the University of California, Lawrence Livermore National Laboratory under contract No. W-7405-Eng-48.

This report has been reproduced directly from the best available copy.

Available electronically at http://www.doc.gov/bridge

Available for a processing fee to U.S. Department of Energy

And its contractors in paper from

U.S. Department of Energy

Office of Scientific and Technical Information

P.O. Box 62

Oak Ridge, TN 37831-0062

Telephone: (865) 576-8401

Facsimile: (865) 576-5728

E-mail: reports@adonis.osti.gov

Available for the sale to the public from

U.S. Department of Commerce

National Technical Information Service

5285 Port Royal Road

Springfield, VA 22161

Telephone: (800) 553-6847

Facsimile: (703) 605-6900

E-mail: orders@ntis.fedworld.gov

Online ordering: http://www.ntis.gov/ordering.htm

\section{OR}

Lawrence Livermore National Laboratory

Technical Information Department's Digital Library

http://www.llnl.gov/tid/Library.html 


\title{
Modeling of Adaptive Optics-based free-space communications systems
}

\author{
Scott C. Wilks*, J. R. Morris, J. M. Brase, S. S. Olivier, J. R. Henderson, C. Thompson, M. Kartz, \\ and A. J. Ruggerio \\ Lawrence Livermore National Laboratory, P. O. Box 808, L290, \\ Livermore, CA 94551
}

\begin{abstract}
We introduce a wave-optics based simulation code written for air-optic laser communications links, that includes a detailed model of an adaptive optics compensation system. We present the results obtained by this model, where the phase of a communications laser beam is corrected, after it propagates through a turbulent atmosphere. The phase of the received laser beam is measured using a Shack-Hartmann wavefront sensor, and the correction method utilizes a MEMS mirror. Strehl improvement and amount of power coupled to the receiving fiber for both $1 \mathrm{~km}$ horizontal and $28 \mathrm{~km}$ slant paths are presented.
\end{abstract}

Keywords: Laser propagation, free space communications, adaptive optics, atmospheric propagation, MEMS mirrors, SATRN

\section{INTRODUCTION}

One of the most desirable features of free space communications is the potential for multi-Gbps data rates. However, a number of limitations due to atmospheric propagation effects that are present on a realistic link make it difficult to achieve these large data rates in practice. One solution is to use Adaptive Optics (AO) to correct the phase of the transmitted beam such that a better focus onto the detector can be achieved, thus increasing the received power, and decreasing the Bit Error Rate (BER). The possibility of using adaptive optics to correct phase aberrations that arise on laser beams as they propagate through the atmosphere has been discussed numerous times. ${ }^{1,2}$ There also exist a set of papers on simulating laser beams propagating through the atmosphere, ${ }^{3,4}$ and another set of papers that deal with the use of adaptive optics in a laser communications system. ${ }^{5,6}$ In this paper, we present results of simulations of a complete adaptive optics system, including wave optics propagation through the atmosphere., This work was done in support of an LLNL program to develop technologies for long-range terrestrial laser communications links known as SATRN ${ }^{7}$ (Secure Air-optic Transport and Routing Network.) In particular, we discuss the salient features of a simulation code developed to help guide the design and implementation of the adaptive optics component of a long-range free space communications link. There are 3 distinct aspects of the code: (1) a wave-optic model of atmospheric propagation of a laser beam through the atmosphere for either short $(\sim 1 \mathrm{~km})$ links or long $(\sim 30 \mathrm{~km})$ links; (2) a complete mathematical model of the adaptive optics components and control system for an 11 x 11 MEMS-based deformable mirror; and (3) wave-optic model for the transport and coupling of the corrected laser light into a 62 micron multi-mode fiber.

Sections 3-5 contain results obtained from the code. The results of this initial idealized model suggest that for a $28 \mathrm{~km}$ link, the use of an $11 \times 11$ MEMS DM mirror may improve the amount of energy that is captured by the receiver and ultimately focused into the receiving fiber for moderate turbulence conditions $\left(\mathrm{C}_{\mathrm{n}}{ }^{2} \sim 5 \times 10^{-14} \mathrm{~m}^{-2 / 3}\right)$ by a factor of 5 . By studying equivalent total $r_{0}$ simulations that include time dependent effects over the $1.3 \mathrm{~km}$ link, we are able to place a lower limit on how fast the system must operate $(\sim 1 \mathrm{kHz}$.) Future studies will include the effects of scintillation on wavefront measurement in the AO system, and strategies for minimizing the deleterious effects that arise because of its presence.

The objective of this report is to demonstrate a set of codes for integrated modeling of air-optic links including adaptive optics. Although the focus of this work is on compensated receivers, the code can also be used to model compensated transmitters. 


\section{ATMOSPHERIC AND ADAPTIVE OPTICS MODELS}

\subsection{Atmospheric propagation models}

We start by initializing a set of phase screens with Kolmogorov turbulence. Each screen represents a length $\delta \mathrm{L}_{Z}$ (typically $100 \mathrm{~m}$ ) of atmosphere, compressed into a thin slab of "equivalent" phase. By including subharmonics, ${ }^{8}$ we can get relatively accurate statistics for the turbulence, ${ }^{9}$ as shown in Fig. 1. The first approximation we make is Taylor's hypothesis, which means that we assume a frozen atmosphere during the laser propagation. This laser light propagates between screens as if in vacuum, using the Fresnel propagation kernel. When light hits a screen, its phase, $\Phi_{L}(\mathrm{x}, \mathrm{y})$, is modified by the effective phase present on the screen, $\Phi_{\mathrm{SCR}}(\mathrm{x}, \mathrm{y})$. By moving the screens transversely (perpendicular to the beam) between discrete laser propagations, we can simulate the effects of the wind during laser propagation. By defining the transmitting laser beam at one end, we can propagate a light wave along a link. An aperture on the final screen represents the receiving telescope, sometimes called the Primary Mirror (PM.)

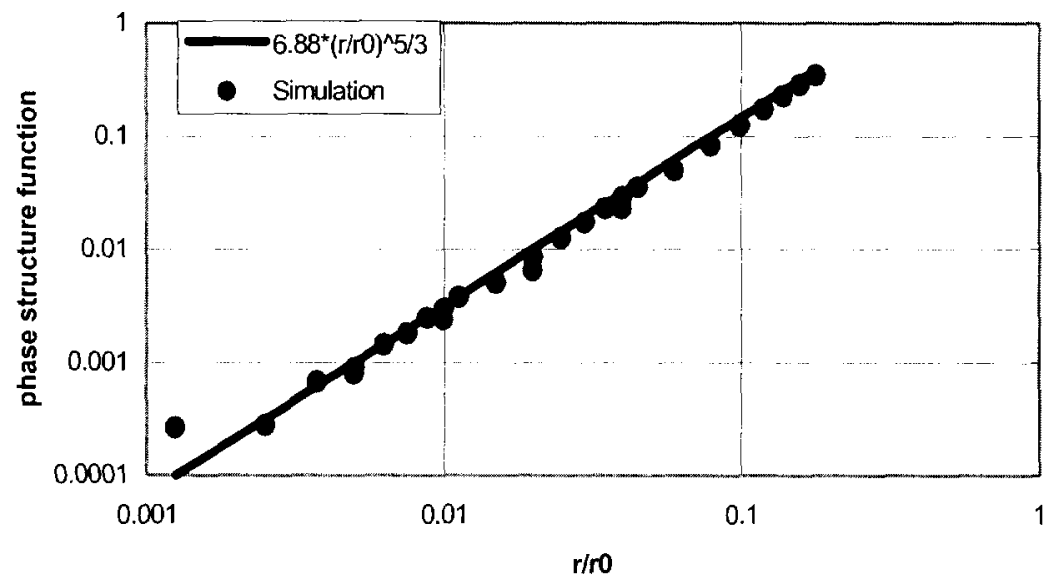

Fig. 1 Phase structure function for the phase screens used in the propagation code for the $1.3 \mathrm{~km}$ link.

It is not feasible to use this brute force approach on the longer link $(28 \mathrm{~km}$.) Because the beam spreads significantly (by a factor of 10) the size and number of screens needed to accurately simulate the propagation in this way becomes prohibitive. For the long link, we employ a technique that uses a Talonov transformation, effectively increasing the grid size as the beam propagates. The wind velocity effect is not currently implemented in the long link simulation. Consequently, multiple runs are made to accumulate statistics, and it is not possible to do temporal studies such as the time response of the $\mathrm{AO}$ system.

\subsection{Adaptive optics model}

We are interested in modeling a particular deformable mirror (DM.) The full aperture of this optic is $3.3 \mathrm{~mm}$, and consists of an array of $12 \times 12$ individually actuated mirrors, each approximately $270 \mu \mathrm{m}$. Fig. 2 shows the geometry used to represent the actuators associated with the DM, along with the mapping of these onto the Hartmann wavefront sensor subapertures. The influence function, which is used to represent the fact that this is a continuous DM, is shown in 
the insert. We are modeling just the inner $11 \times 11$ so that subtleties of edge actuators can be ignored. Also shown is an example phase that is applied to the DM for a particular received beam.

$$
\begin{aligned}
& \text { White = actuators } \\
& \text { Red = subapertures }
\end{aligned}
$$

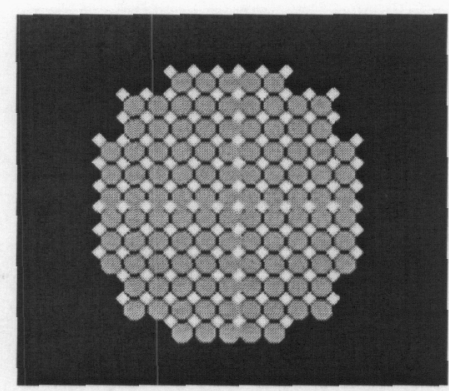

(a)
Influence function

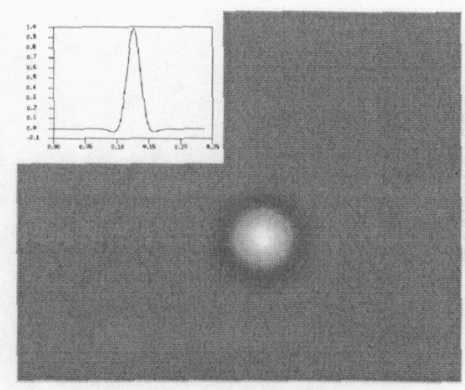

(b)
Example $\phi$ on DM

$$
-15<\phi<5 \mathrm{rad}
$$

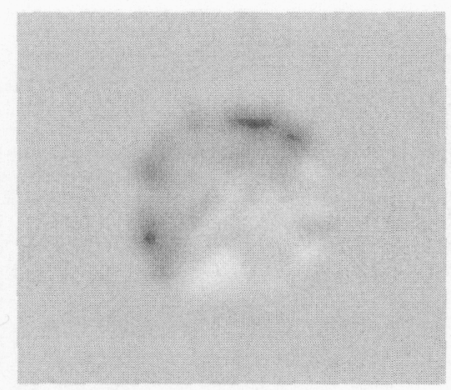

(c)

Fig. 2 (a) Geometries of the actuators associated with deformable mirror, and subapertures associated with the Hartmann sensor. (b) influence function used to model a single element of the continuous face MEMS DM. (c) Example DM phase that is applied to the received wavefront that would result in correction.

We create a wavefront sensor flat reference file by sending in a perfect laser beam, and noting the centroid positions., We then generate the system matrix by pushing each actuator and noting the response, just as in a real AO system, then pseudo-invert to get control matrix. ${ }^{10}$ Once this control matrix is known, it is a simple matter to move the actuators, depending on the position of the Hartmann spots in the subapertures.

Because we include the effects of wind, we can also study temporal aspects of the adaptive optics system. In particular, we can set the time interval between various sensings of the incoming wave, to mimic the time sampling of the actual experimental set-up. In addition, we include a gain in the loop that is typically set to a value of 0.70 .

The performance of an adaptive optics system is typically give by a Strehl ratio, which we take to be defined as the ratio of the peak laser intensity measured on axis, as compared to the theoretical diffraction limited peak intensity that would result for the given receiver aperture. We use this parameter as a means of evaluating the performance of the modeling codes. Typically, we will show tilt-corrected Strehl values, which are the values that will be obtained if a fast tip-tilt mirror is in the system (as in the SATRN experiment that we are modeling.) In addition to this, we also consider the amount of energy that can be focused into a receiver or fiber of a given diameter.. This is the relevant parameter for evaluating the effectiveness of the adaptive optics system in determining the performance of the laser communications system.

\subsection{Fiber Coupling Model}

In the case we are interested in, namely direct coupling of received light to the end of a 62.5-micron multi-mode fiber; the amount of light to be coupled into the fiber is an important quantity for a communications system. For the case of the $28 \mathrm{~km}$ link, we have developed an optical model for the light as it travels from receiver telescope to the fiber. We take the receiving telescope aperture to be $0.3 \mathrm{~m}$, and this light is then sent to a telescope, then to a focusing lens with a focal length of $40 \mathrm{~mm}$, and finally into the end of the multi-mode fiber. This model tells us very precisely, for our experimental set-up, how much of the received power received at the primary mirror gets coupled into the fiber. Section 4 will present results comparing coupling efficiency with and without AO correction. It is important to maximize the amount of energy coupled into this fiber, as a further decrease in power will result once this fiber is coupled to the 8micron fiber (single mode) that is on the receiver side. 


\section{EXAMPLE RESULTS FOR 1.3 KM LINK}

We begin with the results for a $1.3 \mathrm{~km}$ link with an atmosphere chosen such that the total $\mathrm{r}_{0}$ is approximately what might be expected by traveling along a slant path of $28 \mathrm{~km}$ with a horizontally changing $\mathrm{Cn} 2$ profile as discussed in the next section. The diameter of the transmitting and receiving telescope is $0.4 \mathrm{~m}$. The atmosphere was characterized by having a $\mathrm{Cn}^{2}=3 \times 10^{-14} \mathrm{~m}-2 / 3$, and a wind velocity of $3 \mathrm{~m} / \mathrm{s}$ across the direction of the laser beam. The results are shown in Fig. 3. One can see an enhancement of the beam quality of the AO corrected case.
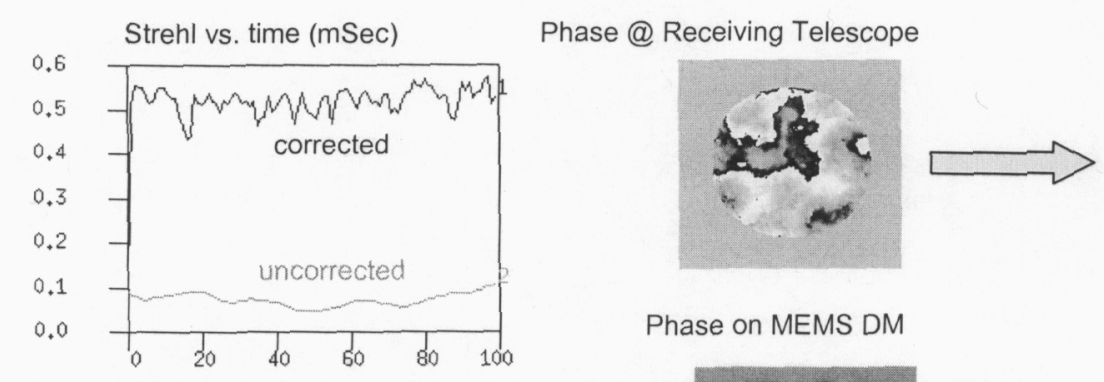

Uncorrected image
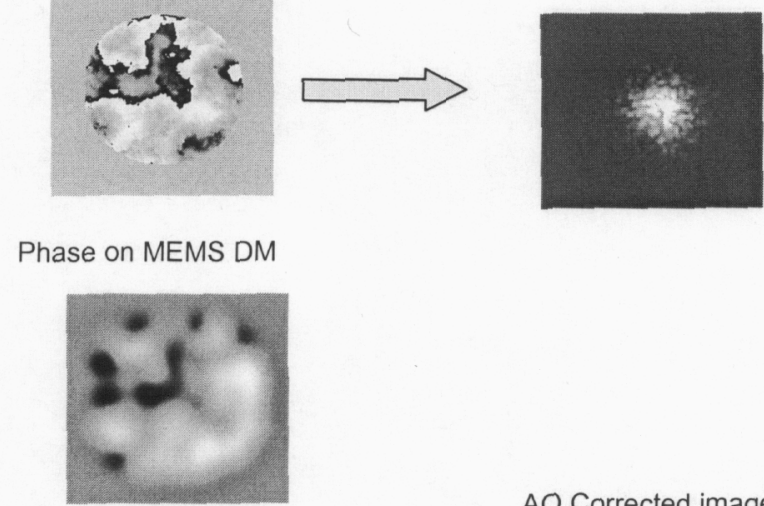

Photon detection
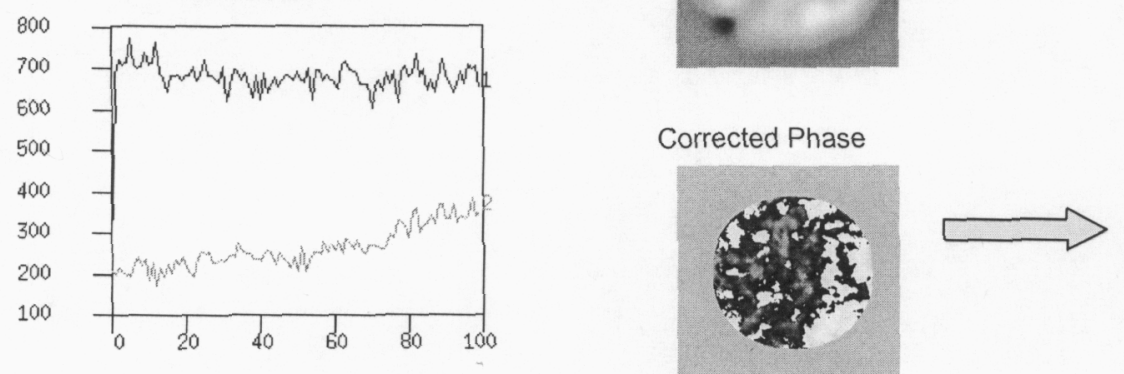

AO Corrected image

Fig. 3 Results from an example $1 \mathrm{~km}$ case, with parameters as quoted in the text.

The best correction that is possible for this case, assuming fitting error is the limit, is a Strehl of 0.68 of what a diffraction limited plane wave system would give. The variance associated with this error is $\sigma^{2} \sim 0.3\left(d / r_{0}\right)^{5 / 3}$, where $d=$ subap size. For the simulation shown in Fig. 3, d/r $r_{0}=1.157$. However, the peak Strehl attainable for the geometry of our finite size transmitter was 0.86 . Therefore, the best corrected Strehl we can expect would be about 0.58 , which is somewhat higher than the 0.52 measured in the simulation, due mainly to temporal response of the system (discussed below.) This is still a factor of approximately 7 greater than the uncorrected Strehl that is obtained by simply focusing the received laser light from the receiver without applying a correction. Note that tilt correction is assumed in these calculations, and is present in the system, in the form of a $300 \mathrm{~Hz}$ fast steering mirror.

Since this simulation system includes wind as it moves the atmospheric phase sheets across the beam, time-dependent phenomena associated with the adaptive optics loop can also be investigated with this tool. In particular, we can study the degradation of the systems ability to focus the light to a tight spot due to the fact that a there is a time delay between the time at which the beam is sensed, and when the correction is applied. This effectively models what is commonly referred to as the bandwidth of the closed loop AO system. Intuitively, one typically takes the atmosphere to be frozen on millisecond time scales. This implies that if one can run at $\sim 1 \mathrm{kHz}$, i.e. measure the wave front at a given instant, and correct the wavefront $1 \mathrm{msec}$ later, the correction applied to the second wavefront should result in nearly perfect correction. This is, for the most part true, until wind speeds become large. In fact a detailed calculation, first derived Greenwood $^{11}$, shows that the degradation in Strehl is given by ${ }^{12}$ 
where the variance is given by

$$
I_{s}(t)=I_{0} e^{-\sigma^{2}}
$$

where $\mathrm{f}_{\mathrm{G}}$ is the Greenwood frequency given by

$$
\sigma^{2}=\left(f_{G} / f_{\text {servo }}\right)^{5 / 3}
$$

$$
f_{G}=0.43 \frac{V_{\text {wind }}}{r_{0}}
$$

and the servo bandwidth is given by

$$
f_{\text {servo }}=\frac{0.3}{\tau_{\text {delay }}}
$$

where $\tau_{\text {delay }}$ is the time lag between measuring the wavefront and applying the correction. By doing the same simulation described above for a number of different wind speeds and time delays (and a slightly worse atmospheric path of $\mathrm{C}_{\mathrm{n}}^{2}=5$ $\left.\mathrm{x} 10^{-14} \mathrm{~m}^{-2 / 3}\right)$ a graph of the effect of bandwidth on the system performance can be generated, as shown in Fig. 4 . We see that for our simulated system, a loop speed of greater than $100 \mathrm{~Hz}$ is desirable (if $\mathrm{V}_{\text {wind }} \sim 2 \mathrm{~m} / \mathrm{s}$ or less), or the $\mathrm{AO}$ correction will be less and less effective. Typical wind speeds for our experimental area are between 1 to $5 \mathrm{~m} / \mathrm{s}$.

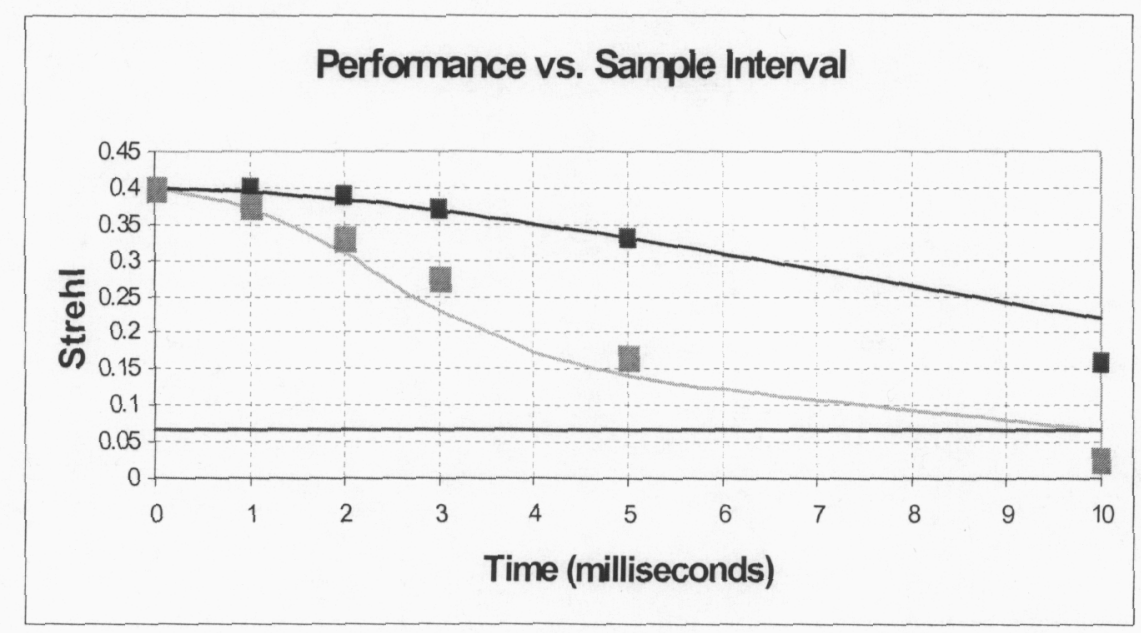

Fig. Degradation of Strehl due to temporal response of the AO system. Dark black curve (points) correspond to predicted (simulated) performance with a wind speed of $2 \mathrm{~m} / \mathrm{s}$. The (lower) gray curve (points) represent predicted (simulated) performance for a wind speed of $5 \mathrm{~m} / \mathrm{s}$. The constant curve at the bottom gives the correct that tilt-only would provide to the system.

\section{EXAMPLE RESULTS FOR 28 KM LINK}

Up to this point, the small screens ( $0.8 \mathrm{~m}$ in transverse dimension, allowing for Nyquist sampling of the received beam) have been adequate for modeling the relatively short link. However, the most important aspect of the SATRN program is the ability to propagate a communications laser over large horizontal and slant distances on the order of several kilometers. Atmospheric screens such as those described above are too large for present computers to yield useful information in a reasonable time scale. Therefore, we resort to using a Talanov transformation for the propagation of the laser through the atmosphere. Basically, a small, tight grid ( 0.8 m, 1024 grids $)$ is used at the transmitter. As the beam propagates, the spacing between the grid points grows, as does the beam size. The beam is free to wander on this grid, and typically much of the energy is spread over a significant part of the grid after propagating through the atmosphere. This is meant to be representative of the test link described in Johnson, et al. ${ }^{7}$ which is approximately 28 kilometers 
along a slant path, from LLNL to a point on a nearby mountain, Mt. Diablo.. The atmospheric turbulence profile for this case is given by

$$
C_{n}^{2}=C_{0}^{2}\left(\frac{h}{h_{0}}\right)^{-4 / 3}
$$

where $h$ is the beam height above ground, and $h_{0}$ is the distance of the transmitter above ground (after Walters and Kunkel. ${ }^{13}$ ) Once the beam makes it to the receiver, we use the identical code as described above to calculate what the AO system will measure and send to the DM. Although we can now get true wave optic propagation through this large amount of atmosphere, because of the complex transformation necessary, we cannot simply "move" the phase screens to mimic the wind. Therefore, we can only do discrete, uncorrelated instances of atmospheres. This simply means that we cannot look at the temporal aspects of AO correction. However, we can still calculate the amount of spatial correction that we can expect over this distance.

We will look at three separate cases: weak, moderate, and strong turbulence. Let's consider the weak case first. In this case, we take the integrated $\mathrm{r}_{0}=12 \mathrm{~cm} @ 1.5$ microns. We find that for this case, the amount of power coupled from the receiver in the fiber is already $90 \%$ without $\mathrm{AO}$. This is due to the fact that the phase at the receiving telescope is relatively smooth to begin with, and the $\mathrm{AO}$ correction does not provide much improvement (increasing only slightly to $91 \%$ ) Fig. 5 shows example intensity and phase plots at the receiver, and at the far field, of the propagated laser beam for moderate turbulence, $\mathrm{r}_{0}=3.03 \mathrm{~cm} @ 1.5$ microns for both corrected and uncorrected light. As can be seen the phase at the receiving telescope has been distorted after propagating the full $28 \mathrm{~km}$. In this case, the AO system calculates the correction to apply to the DM, and this applied phase to the DM can be seen in the figure. Clearly, the phase after the DM is much more flat, than the uncorrected phase. This example yielded an increase in approximately an increase in coupled power of $\sim 2.5$ by using AO correction. Recall that although the effect of scintillation is present in the simulation, it is not accounted for in the wavefront measurement section of the code at this time.
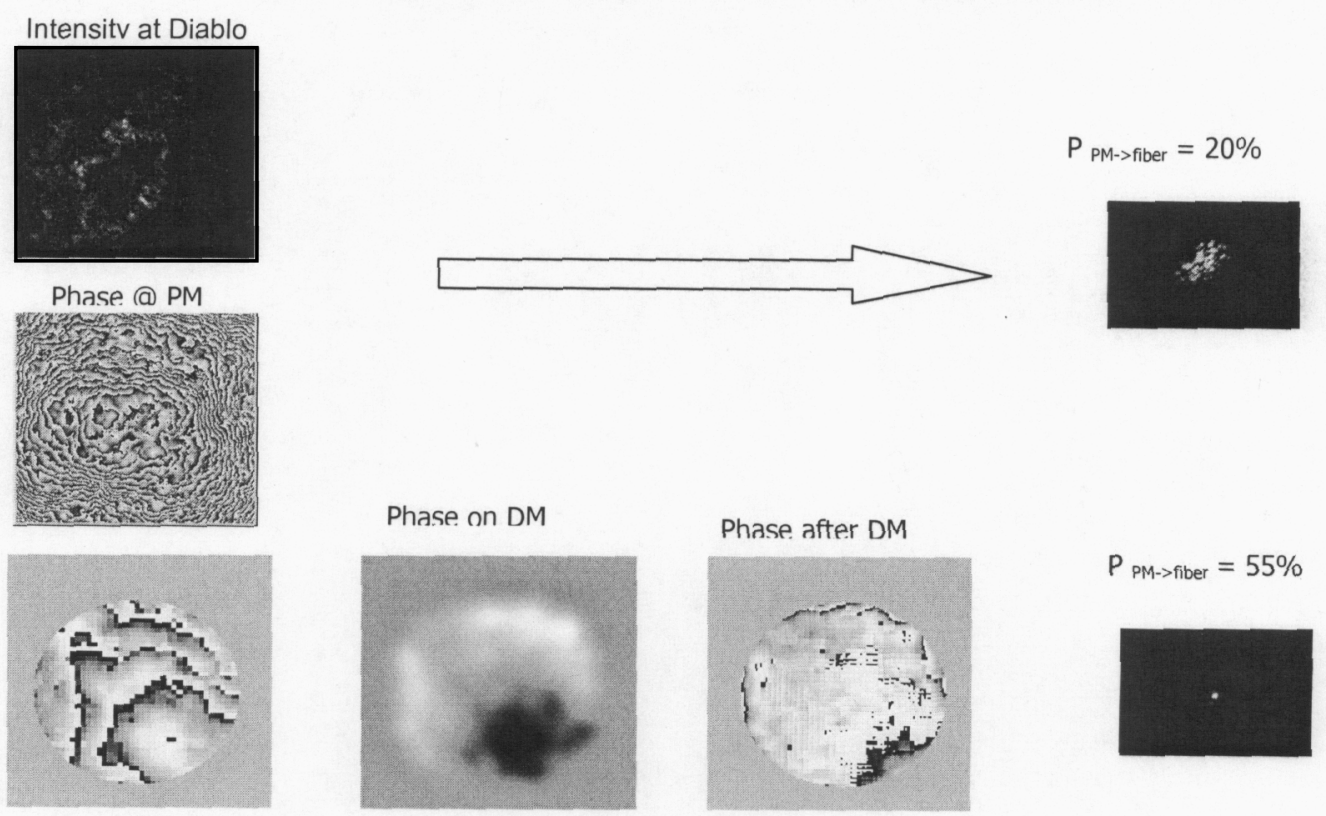

Fig. 5 Example phase and intensity plots of the moderate turbulence case, as well as a few example far-field spots for the AO off case (phase uncorrected) and $\mathrm{AO}$ on (phase after DM) case.

Fig. 6 shows the results of a number of runs done with moderate turbulence, $\mathrm{r}_{0}=3.03 \mathrm{~cm} @ 1.5$ microns. In this case, there is a clear advantage of using AO, if one is concerned with coupling the light directly into a fiber. In addition to the power increase, the stability of the laser power into the fiber, relative to the spread in energies that are obtained if no correction is applied is also noteworthy for communications applications. 


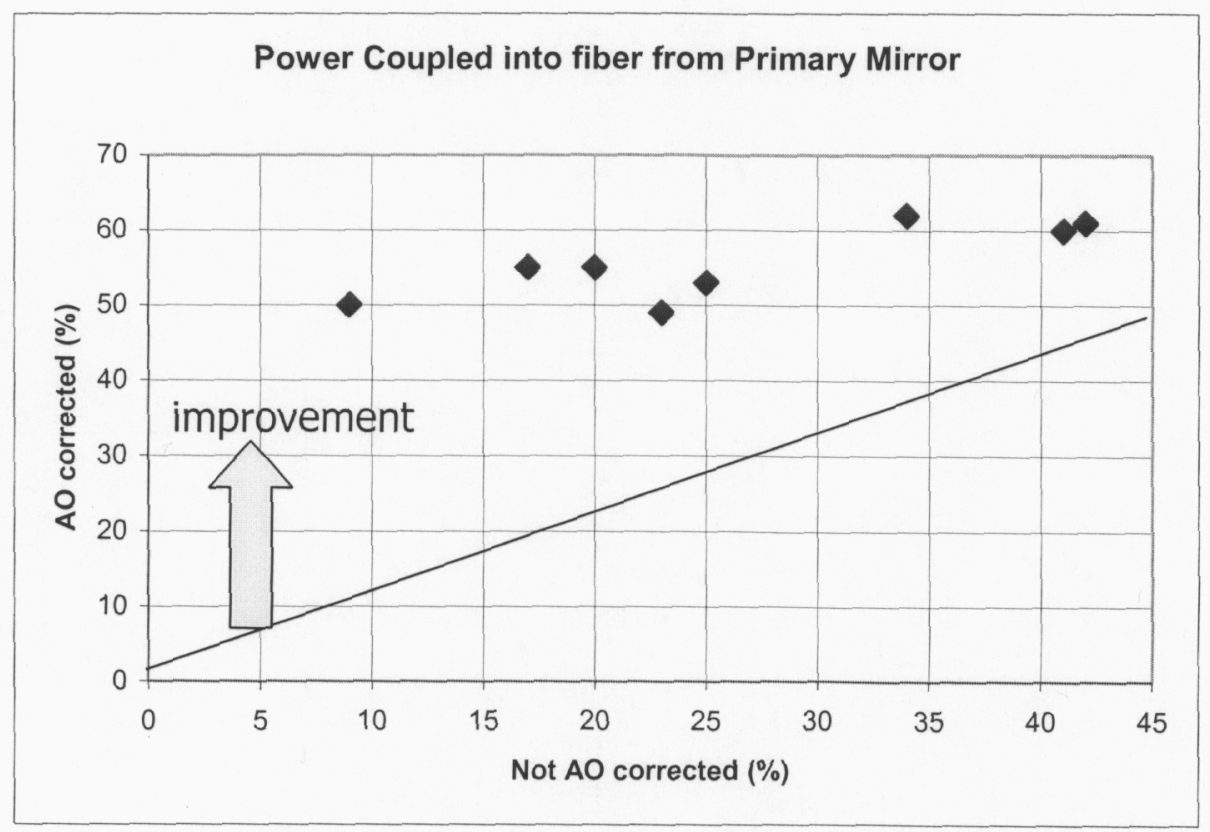

Fig. 6 Amount of power coupled into a 62.5 multimode fiber, relative to the amount captured at the receiver for a number of atmospheric realizations for a $28 \mathrm{~km}$ link with moderate turbulence. All points that lie in the region above the diagonal line represent improvement over an uncorrected receiver.

Finally, we simulate a strong turbulence case $\left(\mathrm{r}_{0}=7 \mu \mathrm{m} @ 1=1.5 \mathrm{~mm}\right)$ for the $28 \mathrm{~km}$ slant path. The received phase and intensity is shown in Fig. 7. Clearly, scintillation is non-negligible in this case, and therefore any linear reconstruction done on this wavefront would be fictitious; in fact, the Rytov variance ${ }^{14}$ is $\sigma_{1}{ }^{2} \sim 0.35$. Wave-front sensing and DM control in strong scintillation has been addressed by both Roggemann and Koivunen, ${ }^{15}$ and more recently by Barchers, Fried and Link. ${ }^{16}$ We plan to implement the algorithms discussed in both of these references as a next step in the development of this model.


Fig. 7 (a) Intensity and (b) phase at receiver plane of laser light after propagating $28 \mathrm{~km}$ in strong scintillation case. Each side is 2 meters in length. 


\section{CONCLUSIONS}

A robust model of an AO system based on a Hartmann WFS and a continuous MEMS DM, along with wave optics propagation through the laser beam, has been developed to model an air-optic communications link.. Initial modeling results suggest that using a $11 \mathrm{X} 11 \mathrm{DM}$ and a $100 \mathrm{~Hz}$ closed loop BW, an increase in power coupling efficiency to a multimode fiber receiver may be possible. Extension of this simple idealized model to include scintillation effects is currently underway.

\section{ACKNOWLEDGEMENTS}

We would like to acknowledge useful conversations with Gary Johnson, Carmen Carrano, Peter Young, Kevin Baker, and Claire Max. This work was performed under the auspices of the U.S. Department of Energy by the University of California, Lawrence Livermore National Laboratory under contract No. W-7405-Eng-48.

\section{REFERENCES}

1. See J. W. Hardy, Adaptive Optics for Astronomical Telescopes, Oxford University Press, Oxford, (1998).

2. R. K. Tyson, Principles of Adaptive Optics, $2^{\text {nd }}$ Edition, Academic Press, Boston, (1997).

3. J. M. Martin and S. Flatte, Applied Optics 27, 2111 (1988).

4. S. Flatte, C. Bracher, and G.-Y. Wang, "Probability-density functions of irradiance for waves in atmospheric turbulence calculated by numerical simulation", J. Opt. Soc. Am. A, 11, 2080 (1994).

5. B. M. Levine, E. A. Martinsen, A. Wirth, A. Jankevics, M. Toledo-Quinones, F. Landers, and T. L. Bruno, "Horizontal Line-of-sight turbulence over near-ground paths and implications for adaptive optics corrections in laser communications", Appl. Opt. 37, 4553, (1998).

6. P. R. Barbier, D. W. Rush, H. Pries, P. Polak-Dingels, and M. L. Plett, "Charactierizaiton of optical wireless link performance", SPIE Conf. On Optical Wireless Communications, Boston, SPIE Vol. 3532, 41 (1998).

7. G. W. Johnson, et. al., "Characterization of Gigabit Ethernet over highly turbulent optical wireless links," Proceedings of SPIE Vol. 482I, Seattle July 2002, paper no. 4821-32

8. E. M. Johansson and D. T. Gavel, "Simulation of Stellar Speckle Imaging", UCRL-JC-115104, (1994).

9. D. L. Fried, JOSA, 55, 1427 (1965).

10. J. M. Brase, J. R. An, K. Avicola, B. V. Beeman, G. L. Berry, B. Johnston, C. Max, J. A. Watson, K. E. Waltjen, "Keck adaptive optics: wavefront control system: architecture and initial laboratory results", Proceedings of the SPIE, 3353, Adaptive Optics System Technologies (1998).

11. Greenwood, "Bandwidth specification for adaptive optics systems," J. Opt. Soc. Am. 67, 174 (1977).

12. This presentation follows that of J. M. Beckers, Ann. Rev. of Astro. and Astrophysics, 31, 13-62 (1993).

13. D. Walters and W. Kunkel, The IR/EO Handbook, Section 2.5 "Models of Optical Turbulence," 211 (1975).

14. For example, in Andrews, Phillips, Hopen Laser Beam Scintillation with Applications, SPIE Press, (2001).

15. M. C. Roggemann and A. C. Koivunen, "Wave-front sensing and deformable-mirror control in strong scintillation," J.Op. Soc. Am. A., 17, 911 (2000).

16. J. D. Barchers, D. L. Fried, and D.J. Link, "Evaluation of the performance of Hartmann sensors in strong scintillation," Applied Optics 41, 1012 (2002). 